\title{
ENTANGLEMENT PROPERTIES OF POSITIVE OPERATORS WITH RANGES IN COMPLETELY ENTANGLED SUBSPACES
}

\author{
R SENGUPTA, ARVIND, AND AJIT IQBAL SINGH
}

\begin{abstract}
We prove that the projection on a completely entangled subspace $\mathcal{S}$ of maximum dimension in a multipartite quantum system obtained by Parthasarathy Par04 is not positive under partial transpose. We next show that several positive operators with range in $\mathcal{S}$ also have the same property. In this process we construct an orthonormal basis of $\mathcal{S}$ and provide a linking theorem to link the constructions of completely entangled subspaces due to Parthasarthy, Bhat and Johnston.
\end{abstract}

\section{INTRODUCTION}

Entanglement is one of the key distinguishing features of quantum mechanics which separates the quantum description of the world from its classical counterpart. Ever since its discovery by Schrödinger [Sch35, Sch36] and its use by Einstein, Podolsky and Rosen [EPR35], the study of entanglement has played a central role in the area of quantum theory and a huge volume of literature is available in this context. In recent years, with the emergence of quantum information where quantum entanglement gets intimately connected to the computational advantage of quantum computers and to the security of quantum cryptographic protocols, its study has become even more important. A detailed discussion on these topics is available in the standard textbook of Nielsen and Chuang [NC10], and a lucid introduction by Parthasarathy [Par06] as well as a rigorous information theoretic account by Wilde [Wil13 are also very useful resources.

Entangled quantum states are those for which it is not possible to imagine the physical reality of a composite quantum system as two separate entities, even when there is no active interaction between the two subsystems. In general linear combinations of entangled states need not be entangled, however, there have been constructions of subspaces where every state in the subspace is entangled. The first such construction was through the unextendable product basis(UPB) by Bennett et. al. [BDM ${ }^{+}$99, and further extended by DiVincenzo et. al. $\left[\mathrm{DMS}^{+} 03\right.$. More recently, Parthasarathy Par04, Bhat Bha06, and Johnston [Joh13] have, by their own different methods, constructed completely entangled subspaces $\mathcal{S}$ of maximum possible dimension in the state space of multipartite quantum systems of finite dimensions. In such a subspace every state in the subspace is entangled.

In our work we focus on projection operators on such completely entangled subspaces. We give a linking theorem which links the constructions of Parthasarathy, Bhat and Johnston. Parthasarathy [Par04] gave an orthonormal basis for $\mathcal{S}$ for the bipartite case of equal dimensions. We develop a method for construction of an orthonormal basis for the space $\mathcal{S}$ in the general case. Further, we construct the (orthogonal) projection on the space $\mathcal{S}$ and show that it is not

The authors thank K. R. Parthasarathy for insightful discussion, critical comments and useful suggestions for the paper. They also thank Nathaniel Johnston for his careful reading of the manuscript, critical comments, corrections and suggestions which improved the paper. Ajit Iqbal Singh thanks Indian National Science Academy (INSA) for INSA Honorary Scientist position and Indian Statistical Institute for invitation to visit the institute under the scheme together with excellent research facilities and stimulating atmosphere. 
positive under partial transpose at any level $j$. The proof utilizes the orthonormal basis for $\mathcal{S}$ that we develop. Finally, we show that a large class of positive operators with range in $\mathcal{S}$ are not positive under partial transpose at level $j$. This extends a substantial part of Johnston's result for the bipartite case to the multipartite case by an altogether different method.

The material in this paper is organized as follows: We begin Section 2 with the basics of quantum entanglement. We then describe the constructions of completely entangled subspaces by Parthasarthy, Bhat and Johnston. Next we give a theorem linking these three constructions. Then we give a construction procedure of an orthonormal basis for theses spaces. In Section 3 we discuss our main results regarding the entanglement properties of projection operators on completely entangled subspace as also of certain positive operators with support in this space. Section 4 offers some concluding remarks.

\section{Completely entangled spaces}

We begin with some well known concepts and results.

\subsection{Entanglement.}

Definition 2.1. A finite dimensional quantum system is described by a finite dimensional complex Hilbert space $\mathcal{H}$. A Hermitian, positive semidefinite operator $\rho \in \mathcal{L}(\mathcal{H})$, the algebra of linear operators on $\mathcal{H}$ to itself, with unit trace is said to be a state of the system $\mathcal{H}$. Rank 1 states are called pure states. A pure state can be written as an outer product $\rho=|\psi\rangle\langle\psi|$ where $|\psi\rangle \in \mathcal{H}$ and $\langle\psi \mid \psi\rangle=1$.

Definition 2.2. A state $\rho$ acting on a bipartite system $\mathcal{H}_{1} \otimes \mathcal{H}_{2}$ is said to be separable if it can be written as

$$
\rho=\sum_{j=1}^{m} p_{j} \rho_{j}^{(1)} \otimes \rho_{j}^{(2)}, \quad p_{j}>0, \quad \sum_{j=1}^{m} p_{j}=1,
$$

where $\rho_{j}^{(1)}$ and $\rho_{j}^{(2)}$ are states in the system $\mathcal{H}_{1}$ and $\mathcal{H}_{2}$ respectively.

Definition 2.3. A state is said to be entangled, if it is not separable by the above definition. Entangled states can be pure or mixed. For an entangled pure state $\rho=|\psi\rangle\langle\psi|,| \psi\rangle$ is called an entangled (unit) vector and any non-zero multiple of $|\psi\rangle$ is called an entangled vector.

If the state is pure and separable, then it can be written in the form $|\psi\rangle=\left|\psi_{1}\right\rangle \otimes\left|\psi_{2}\right\rangle$, and hence $\rho=\left|\psi_{1}\right\rangle\left\langle\psi_{1}|\otimes| \psi_{2}\right\rangle\left\langle\psi_{2}\right|$. If we take partial trace with respect to any of the subsystems, say $\mathcal{H}_{2}$, then we get a pure state $\operatorname{Tr}_{\mathcal{H}_{2}} \rho=\left|\psi_{1}\right\rangle\left\langle\psi_{1}\right|$ as the reduced density matrix. On the other hand, for an entangled pure state we always get a mixed state after a partial trace. Hence, a pure state is separable if and only if the reduced density matrices are of rank one. This method does not work for mixed states.

We also consider multi-partite quantum systems, where the state space given by $\mathcal{H}=\mathcal{H}_{1} \otimes$ $\cdots \otimes \mathcal{H}_{k}$; or in short, $\bigotimes_{j=1}^{k} \mathcal{H}_{j}$. A product vector in this multipartite system space is written as $\left|x_{1}\right\rangle \otimes \cdots \otimes\left|x_{k}\right\rangle$, with $\left|x_{j}\right\rangle \in \mathcal{H}_{j}$ or as $\left|x_{1}, \cdots, x_{k}\right\rangle$ or in short as $\bigotimes_{j=1}^{k}\left|x_{j}\right\rangle$. The state of the system $\mathcal{H}$ can be entangled or separable. An important open problem in the field is to determine whether an arbitrary state $\rho$ of an arbitrary quantum system $\mathcal{H}$, is entangled or separable. For further details regarding entanglement we refer the survey article written by Horodecki et. al. [HHHH09]. 
For general states, a very important one way condition to check entanglement is by using partial transpose (PT). If a quantum state becomes non-positive after PT then it is called NPT and if it remains positive after partial transpose it is called PPT. NPT states are definitely entangled and separable states are definitely PPT while PPT states can be entangled or separable. PPT entangled states are also called bound entangled states and their characterization into entangled and separable is a major open issue in the field. Checking PPT condition is also known as the 'Peres test' because of the significant work by Peres [Per96]. As remarked by DiVincenzo et. al. [DMS ${ }^{+}$03], in the case of multipartite systems, the PPT condition can not be used directly. We can check the PPT property under every possible bipartite partitioning of the state. We discuss this process in some detail because of its use in our work.

Definition 2.4. Let, for $1 \leq j \leq k,\left\{\left|p_{j}\right\rangle: p_{j}=0,1, \cdots, \operatorname{dim}\left(\mathcal{H}_{j}\right)-1\right\}$ be an orthonormal basis in $\mathcal{H}_{j}$. Let $\rho \in \mathcal{L}\left(\mathcal{H}_{1} \otimes \cdots \otimes \mathcal{H}_{k}\right)$ be an operator. Then $\rho$ can be expressed in the form

$$
\rho=\sum_{p_{1}, q_{1}=0}^{\operatorname{dim} \mathcal{H}_{1}-1} \cdots \sum_{p_{k}, q_{k}=0}^{\operatorname{dim} \mathcal{H}_{k}-1} \rho_{p_{1}, \cdots, p_{k} ; q_{1}, \cdots, q_{k}}\left|p_{1}, \cdots, p_{k}\right\rangle\left\langle q_{1}, \cdots, q_{k}\right| .
$$

The partial transpose of $\rho$, with respect to the $j$ th system, is given by

$$
\rho^{P T_{j}}=\sum_{p_{1}, q_{1}=0}^{\operatorname{dim} \mathcal{H}_{1}-1} \cdots \sum_{p_{k}, q_{k}=0}^{\operatorname{dim} \mathcal{H}_{k}-1} \rho_{p_{1}, \cdots, p_{k} ; q_{1}, \cdots, q_{k}}
$$

If for a state $\rho, \rho^{P T_{j}}$ is positive, then $\rho$ is said to be positive under partial transpose at the $j$ th level, in short, $P P T_{j}$. If a state $\rho$ is not $P P T_{j}$, then it is said to be not positive under partial transpose at the $j$ th level, in short, $N P T_{j}$.

\section{Remark 2.1.}

(i) It is a fact that the property $P P T_{j}$ is independent of the choice of orthonormal basis in $\mathcal{H}_{j}$.

(ii) In case of any bipartite system $\rho$, it is said to be PPT if it is $P P T_{1}$ or $P P T_{2}$ (in this case $P P T_{1}$ implies $P P T_{2}$ and vice versa).

(iii) Woronowicz [Wor76] showed that, a state in $\mathbb{C}^{2} \otimes \mathbb{C}^{2}, \mathbb{C}^{2} \otimes \mathbb{C}^{3}$ or $\mathbb{C}^{3} \otimes \mathbb{C}^{2}$ is separable if and only if it is PPT. For higher dimensions, PPT is necessary, but not sufficient for separability and there are examples of entangled states which are PPT. First examples of such states were constructed by Choi [Cho80] for $3 \otimes 3$, Woronowicz [Wor76] for $2 \otimes 4$ and later by Størmer Stø82] for $3 \otimes 3$.

Definition 2.5. For any proper subset $E$ of $\{1,2, \cdots, k\}$ and its complement $E^{\prime}$ in $\{1, \cdots, k\}$ let $\mathcal{H}(E)=\bigotimes_{j \in E} \mathcal{H}_{j}$ and $\mathcal{H}\left(E^{\prime}\right)=\bigotimes_{j \in E^{\prime}} \mathcal{H}_{j}$. Then $\mathcal{H}=\mathcal{H}(E) \otimes \mathcal{H}\left(E^{\prime}\right)$. Any such decomposition is called a bipartite cut. A state $\rho \in \mathcal{H}$ is said to be positive under partial transpose, in short, PPT if it is PPT under any bipartite cut.

Remark 2.2. Obviously if $\rho$ is PPT then $\rho$ is PPT for each $j$; all we need to do is is to take $E=\{j\}$. In other words, if $\rho$ is $N P T_{j}$ for some $j$, then it is NPT.

2.2. Unextendable product bases. One well studied way to construct PPT entangled states was given by Bennett et. al. [BDM$\left.{ }^{+} 99\right]$ by using unextendable product basis. 
Definition 2.6. An incomplete set of product vectors $\mathcal{B}$ in the Hilbert space $\mathcal{H}=\bigotimes_{j=1}^{k} \mathcal{H}_{j}$ is called unextendable if the space $\langle\mathcal{B}\rangle^{\perp}$ does not contain any product vector. The vectors in the set $\mathcal{B}$ are usually taken as orthonormal and are called unextendable product bases, abbreviated as $U P B$.

To avoid trivialities, we assume $\operatorname{dim} \mathcal{H}_{j}=d_{j} \geq 2$. Let $D=d_{1} d_{2} \cdots d_{k}$. Bennett et. al. $\left.\mathrm{BDM}^{+} 99\right]$ gave three examples of UPB for bipartite and tripartite systems namely, PYRAMID, TILES and SHIFT. We state the key theorem of Bennett et. al. [BDM ${ }^{+}$99] which allows one to construct PPT entangled states from UPB and which is relevant to this paper.

Theorem A. [BDM $\left.{ }^{+99}\right]$ If in the Hilbert space $\mathcal{H}=\bigotimes_{j=1}^{k} \mathcal{H}_{j}$ of dimension $D=d_{1} \cdots d_{k}$, as above, there is a mutually orthonormal set of unextendable product basis : $\left\{\left|\psi_{s}\right\rangle: s=1, \cdots, d\right\}$, then the state

$$
\rho=\frac{1}{D-d}\left(I_{D}-\sum_{s=1}^{d}\left|\psi_{s}\right\rangle\left\langle\psi_{s}\right|\right),
$$

where $I_{D}$ is the identity operator on $\mathcal{H}$, is an entangled state which is PPT.

The proof depends on the orthogonality of the basis vectors $\left|\psi_{s}\right\rangle$.

The above theory was further extended by DiVincenzo et. al. [DMS ${ }^{+}$] $]$to include generalizations of the earlier examples to multipartite systems and a complete characterization of UPB in $\mathbb{C}^{3} \otimes \mathbb{C}^{3}$. There is a large volume of literature in this area. Recently, Johnston has given explicit computation of four qubit UPB [Joh14].

2.3. Entangled subspaces. Let $\mathcal{H}=\mathcal{H}_{1} \otimes \cdots \otimes \mathcal{H}_{k}$, where for $1 \leq j \leq k, \mathcal{H}_{j}=\mathbb{C}^{d_{j}}$ for some $d_{j}<\infty$ as above. Wallach Wal02] considered the question of the maximal possible dimension of a subspace $\mathcal{S}$ of $\mathcal{H}$ where each nonzero vector is an entangled state. He called such subspaces entangled subspaces, as they do not contain any nonzero product vector. He showed that

Theorem B. Wal02] The dimension of a subspace, where each vector is entangled, is $\leq$ $d_{1} \cdots d_{k}-\left(d_{1}+\cdots+d_{k}\right)+k-1$. Furthermore, this upper bound is attained.

2.4. Parthasarathy's construction. Parthasarathy Par04 gave an explicit construction of such entangled subspaces where the maximal dimension is attained. We note that Parthasarathy calls such subspaces completely entangled subspaces. Let $\mathcal{H}=\mathcal{H}_{1} \otimes \cdots \otimes \mathcal{H}_{k}$ be as above. Let $\lambda \in \mathcal{C}$. For $1 \leq j \leq k$, let

$$
v_{\lambda, j}=\left(\begin{array}{c}
1 \\
\lambda \\
\lambda^{2} \\
\vdots \\
\lambda^{d_{j}-1}
\end{array}\right) \equiv \sum_{x=0}^{d_{j}-1} \lambda^{x}|x\rangle
$$

where $\left\{|x\rangle: x=0,1, \cdots, d_{j}-1\right\}$ is the standard basis of $\mathcal{H}_{j}=\mathbb{C}^{d_{j}}$. Set

$$
\left|v_{\lambda}\right\rangle \equiv v_{\lambda, 1} \otimes \cdots \otimes v_{\lambda, k}=\bigotimes_{j=1}^{k} v_{\lambda, j}
$$

Set $N=\sum_{j=1}^{k}\left(d_{j}-1\right)=\sum_{j=1}^{k} d_{j}-k$. Choose any $(N+1)$ distinct complex numbers $\lambda_{0}, \lambda_{1}, \cdots, \lambda_{N}$ and denote the linear span of $\left\{v_{\lambda_{n}}: 0 \leq n \leq N\right\}$ by $\mathcal{F}$, i.e. $\mathcal{F}=\left\langle v_{\lambda_{n}}: 0 \leq n \leq\right.$ $N\rangle$. Then $\left\{v_{\lambda_{n}}: 0 \leq n \leq N\right\}$ is a basis of $\mathcal{F}$. Consider the subspace $\mathcal{S}=\mathcal{F}^{\perp}$. 
It has been shown in $\mathrm{Par04}$ that the space $\mathcal{S}$ does not contain any product vector and is of dimension $M=d_{1} \cdots d_{k}-\left(d_{1}+\cdots+d_{k}\right)+k-1$.

Simple computations show that the basis vectors of $\mathcal{F}$ need not all be orthogonal, but certain subspaces of $\mathcal{F}$ can contain orthonormal basis of product vectors.

Another strong point in this paper is an explicit construction of an orthonormal basis for $\mathcal{S}$ in the case $k=2, d_{1}=d_{2}$. We shall come back to this later in $\$ 2.7$ below.

2.5. Bhat's construction [Bha06]. For notational convenience, he starts with an infinite dimensional space with an orthonormal basis $\left\{e_{0}, e_{1}, \cdots\right\}$ and identifies $\mathcal{H}_{r}=\left\langle\left\{e_{0}, \cdots, e_{d_{r}-1}\right\}\right\rangle, 1 \leq$ $r \leq k$, and sets $\mathcal{H}=\mathcal{H}_{1} \otimes \cdots \otimes \mathcal{H}_{k}$.

Let $N=\sum_{r=1}^{k}\left(d_{r}-1\right)$. For $0 \leq n \leq N$, let $\mathcal{I}_{n}=\left\{\mathbf{i}=\left(i_{r}\right)_{r=1}^{k}, 0 \leq i_{r} \leq d_{r}-1\right.$ for $1 \leq$ $\left.r \leq k, \sum_{r=1}^{k} i_{r}=n\right\}$. Let $\mathcal{I}=\bigcup_{n=0}^{N} \bar{I}_{n}$. For $\mathbf{i} \in \mathcal{I}$, let $e_{\mathbf{i}}=\bigotimes_{r=1}^{k} e_{i_{r}}$. For $0 \leq n \leq N$, let $\mathcal{H}^{(n)}=\left\langle\left\{e_{\mathbf{i}}: \mathbf{i} \in \mathcal{I}_{n}\right\}\right\rangle$. Then $\left\{e_{\mathbf{i}}: \mathbf{i} \in \mathcal{I}_{n}\right\}$ is an orthonormal basis for $\mathcal{H}^{(n)}$. Further, $\mathcal{H}=\bigoplus_{n=0}^{N} \mathcal{H}^{(n)}$ and $\left\{e_{\mathbf{i}}: \mathbf{i} \in \mathcal{I}\right\}$ is an orthonormal basis for $\mathcal{H}$. Let $0 \leq n \leq N$. Let $u_{n}=\sum_{\mathbf{i} \in \mathcal{I}_{n}} e_{\mathbf{i}}$. Let $\mathcal{T}^{(n)}=\mathbb{C} u_{n}$, then $\mathcal{H}^{(n)}=\mathcal{S}^{(n)} \bigoplus T^{(n)}$, where

$$
\mathcal{S}^{(n)}=\operatorname{span}\left\{e_{\mathbf{i}}-e_{\mathbf{j}}: \mathbf{i}, \mathbf{j} \in \mathcal{I}_{n}\right\}
$$

Clearly $\mathcal{S}^{(n)}$ is also equal to the set of all the sums $\sum_{\mathbf{i} \in \mathcal{I}_{n}} \alpha_{\mathbf{i}} e_{\mathbf{i}}$ such that $\sum_{\mathbf{i} \in \mathcal{I}_{n}} \alpha_{\mathbf{i}}=0$. Further, $\mathcal{S}^{(0)}=\{0\}=\mathcal{S}^{(N)}$. Let $\mathcal{T}=\bigoplus_{n=0}^{N} \mathcal{T}^{(n)}$ and $\mathcal{S}_{B}=\bigoplus_{n=0}^{N} \mathcal{S}^{(n)}$, which is the same as $\bigoplus_{n=1}^{N-1} \mathcal{S}^{(n)}$. Then $\mathcal{S}_{B}^{\perp}=\mathcal{T}$ and $\mathcal{H}=\mathcal{S}_{B} \oplus \mathcal{T}$.

Theorem C. [Bha06] $\mathcal{S}_{B}$ is a completely entangled subspace of maximal dimension.

\section{Remark 2.3.}

(i) We note that for $\lambda \in \mathbb{C}$,

$$
\begin{aligned}
\left|z^{\lambda}\right\rangle & \equiv\left(\sum_{j_{1}=0}^{d_{1}-1} \lambda^{j_{1}} e_{j_{1}}\right) \otimes \cdots \otimes\left(\sum_{j_{k}=0}^{d_{k}-1} \lambda^{j_{k}} e_{j_{k}}\right) \\
& =\sum_{n=0}^{N} \lambda^{n}\left(\sum_{\mathbf{i} \in \mathcal{I}_{n}} e_{\mathbf{i}}\right) \\
& =\sum_{n=0}^{N} \lambda^{n} u_{n} .
\end{aligned}
$$

(ii) We now consider $\mathcal{H}_{r}$ 's as subspaces of $\mathbb{C}^{\delta}$, with $\delta=\max _{j=1}^{k} d_{j}$ and $e_{s} \equiv|s\rangle$ for $1 \leq s \leq \delta$. So we can identify $\left|v_{\lambda}\right\rangle$ and $\left|z^{\lambda}\right\rangle$. Let $\lambda_{n}, 0 \leq n \leq N$ be distinct complex numbers as in \$2.4. Then $\left\{\left|v_{\lambda_{n}}\right\rangle: 0 \leq n \leq N\right\}$ is a linearly independent subset of $\mathcal{T}$. So $\mathcal{F}=\mathcal{T}$. This also shows that $\mathcal{F}$ is independent of the choice of complex numbers. Thus

$$
\mathcal{S}=\mathcal{F}^{\perp}=\mathcal{T}^{\perp}=\mathcal{S}_{B}
$$

Theorem D. [Bha06] The set of product vectors in $\mathcal{S}^{\perp}=\mathcal{T}$ is

$$
\left\{c\left|z^{\lambda}\right\rangle: c \in \mathbb{C}, \lambda \in \mathbb{C} \cup\{\infty\}\right\}
$$

where $\left|z^{\infty}\right\rangle=\bigotimes_{r=1}^{k} e_{d_{r}-1}$ 
2.6. Johnston's construction [Joh13]. Johnston concentrated on constructing a completely entangled subspace $\mathcal{S}_{J}$ of $\mathbb{C}^{d_{1}} \otimes \mathbb{C}^{d_{2}}$ of dimension $\left(d_{1}-1\right)\left(d_{2}-1\right)$ for bipartite systems such that every density matrix with range contained in it is NPT. In the notation of Subsections 2.4 and 2.5 .

$$
\mathcal{S}_{J}=\left\langle\left\{w_{x, y}=|x\rangle \otimes|y+1\rangle-|x+1\rangle \otimes|y\rangle: 0 \leq x \leq d_{1}-2,0 \leq y \leq d_{2}-2\right\}\right\rangle .
$$

We end this subsection with our theorem which establishes an interesting and useful link between different constructions of completely entangled subspaces.

Theorem 2.1. For the bipartite case, the completely entangled spaces $\mathcal{S}, \mathcal{S}_{B}$ and $\mathcal{S}_{J}$ can be identified with each other.

Proof. In view of Remark 2.3 and the discussion in this section, we only need to note that for $0 \leq x \leq d_{1}-2$ and $0 \leq y \leq d_{2}-2, w_{x, y} \in \mathcal{S}^{(x+y+1)}$. Thus $\mathcal{S}_{J} \subseteq \mathcal{S}_{B}$. But $\operatorname{dim} \mathcal{S}_{B}=$ $\left(d_{1}-1\right)\left(d_{2}-1\right)=\operatorname{dim} \mathcal{S}_{J}$. Hence $\mathcal{S}_{B}=\mathcal{S}_{J}$.

2.7. Parthasarathy's orthonormal basis for $\mathcal{S}$ for bipartite case of equal dimensions Par04]. We need the following explicit construction of the orthonormal basis $\mathcal{B}$ of $\mathcal{S}$ given in [Par04] for the bipartite case $\mathcal{H}=\mathcal{H}_{1} \otimes \mathcal{H}_{2}$, with $d_{1}=d_{2}=\nu$, say.

(a) Antisymmetric vectors:

$$
\left|a_{x, y}\right\rangle=\frac{1}{\sqrt{2}}(|x y\rangle-|y x\rangle), 0 \leq x<y \leq \nu-1 .
$$

(b) For $2 \leq n \leq \nu-1$ and $n$ even, vectors of the forms :

$$
\begin{aligned}
\left|b_{0}^{n}\right\rangle & =\frac{1}{\sqrt{n(n+1)}}\left(\sum_{m=0}^{\frac{n}{2}-1}(|m, n-m\rangle+|n-m, m\rangle)-n\left|\frac{n}{2}, \frac{n}{2}\right\rangle\right), \quad \text { and } \\
\left|b_{p}^{n}\right\rangle & =\frac{1}{\sqrt{n}} \sum_{m=0}^{\frac{n}{2}-1} \exp \left(\frac{4 \pi \imath m p}{n}\right)(|m, n-m\rangle+|n-m, m\rangle), \quad 1 \leq p \leq \frac{n}{2}-1 .
\end{aligned}
$$

(c) For $2 \leq n \leq \nu-1$ and $n$ odd, vectors of the form:

$$
\left|b_{p}^{n}\right\rangle=\frac{1}{\sqrt{n+1}} \sum_{m=0}^{\frac{n-1}{2}} \exp \left(\frac{4 \pi \imath m p}{n+1}\right)(|m, n-m\rangle+|n-m, m\rangle), \quad 1 \leq p \leq \frac{n-1}{2} .
$$

(d) For $\nu \leq n \leq 2 \nu-4$ and $n$ even, vectors of the form:

$$
\begin{array}{r}
\left|b_{0}^{n}\right\rangle=\frac{1}{\sqrt{(2 \nu-2-n)(2 \nu-1-n)}}\left(\sum_{m=0}^{\frac{2 \nu-2-n}{2}-1}(|n-\nu+m+1, \nu-m-1\rangle\right. \\
\left.+|\nu-m-1, n-\nu+m+1\rangle)-(2 \nu-2-n)\left|\frac{n}{2}, \frac{n}{2}\right\rangle\right), \quad \text { and } \\
\left|b_{p}^{n}\right\rangle=\frac{1}{\sqrt{2 \nu-2-n}} \sum_{m=0}^{\frac{2 \nu-2-n}{2}-1} \exp \left(\frac{4 \pi \imath m p}{2 \nu-2-n}\right)(|n-\nu+m+1, \nu-m-1\rangle \\
+|\nu-m-1, n-\nu+m+1\rangle), \quad 1 \leq p \leq \frac{2 \nu-2-n}{2}-1 .
\end{array}
$$


(e) For $\nu \leq n \leq 2 \nu-4$ and $n$ odd, vectors of the form:

$$
\begin{array}{r}
\left|b_{p}^{n}\right\rangle=\frac{1}{\sqrt{2 \nu-1-n}} \sum_{m=0}^{\frac{2 \nu-1-n}{2}-1} \exp \left(\frac{4 \pi \imath m p}{2 \nu-1-n}\right)(|n-\nu+m+1, \nu-m-1\rangle \\
+|\nu-m-1, n-\nu+m+1\rangle), \quad 1 \leq p \leq \frac{2 \nu-1-n}{2}-1 .
\end{array}
$$

\section{Remark 2.4.}

(i) An interesting aspect of $\mathcal{B}$ is that for $1 \leq n \leq 2 \nu-3, \mathcal{B}_{n}=\mathcal{B} \cap \mathcal{S}^{(n)}$ is an orthonormal basis for $\mathcal{S}^{(n)}$.

(ii) $\mathcal{B}_{1}=\left\{\left|a_{0,1}\right\rangle\right\}$ and $\mathcal{B}_{2 \nu-3}=\left\{\left|a_{\nu-2, \nu-1}\right\rangle\right\}$.

(iii) For $1 \leq g \leq \nu-2,\left|f_{g}\right\rangle=|g\rangle \otimes|g\rangle$ occurs as a summand of exactly one vector in $\mathcal{B}$. Further, $\left|f_{\nu-1}\right\rangle=|\nu-1\rangle \otimes|\nu-1\rangle$ does not occur as a summand of vectors in $\mathcal{B}$.

(iv) Let $\mathbb{F}: \mathcal{H}_{1} \otimes \mathcal{H}_{2} \rightarrow \mathcal{H}_{2} \otimes \mathcal{H}_{1}$ be the linear operator, called FLIP or SWAP, satisfying $\mathbb{F}(|\xi\rangle \otimes|\eta\rangle)=|\eta\rangle \otimes|\xi\rangle$ for $|\xi\rangle \in \mathcal{H}_{1}$ and $|\eta\rangle \in \mathcal{H}_{2}$. Then $\mathbb{F}\left(\left|a_{x, y}\right\rangle\right)=-\left|a_{x, y}\right\rangle$, whereas $\mathbb{F}\left(\left|b_{p}^{n}\right\rangle\right)=\left|b_{p}^{n}\right\rangle ;\left|a_{x, y}\right\rangle$ and $\left|b_{p}^{n}\right\rangle$ are as above.

2.8. Bhat's orthonormal basis for $\mathcal{S}$. Bhat [Bha6] indicated how to construct an orthonormal basis for $\mathcal{S}$. He has also given expressions for dimensions of $\mathcal{H}^{(n)}$ viz., $\left|\mathcal{I}_{n}\right|$ for $1 \leq n \leq N$. In fact, $\mathcal{I}_{n}=$ the coefficient of $x^{n}$ in the polynomial $p(x)=\prod_{r=1}^{k}\left(1+x+\cdots+x^{d_{r}-1}\right)=$ number of partitions of $n$ into $\left(i_{1}, \cdots, i_{k}\right)$ with $0 \leq i_{r} \leq d_{r}-1$ for $1 \leq r \leq k$. For instance, for $k=2, d_{1} \leq d_{2}$,

$$
\left|\mathcal{I}_{n}\right|= \begin{cases}n+1 & \text { for } \quad 0 \leq n \leq d_{1}-1 \\ d_{1} & \text { for } \quad d_{1}-1<n \leq d_{2}-1 \\ d_{1}+d_{2}-(n+1) & \text { for } \quad d_{2}-1<n \leq d_{1}+d_{2}-2\end{cases}
$$

If $d_{i}=2$ for all $i$, then $\left|\mathcal{I}_{n}\right|=\left(\begin{array}{l}k \\ n\end{array}\right), 0 \leq n \leq k$.

2.9. Two useful techniques. We now display techniques to be used in constructing an orthonormal basis for the general bipartite and multipartite case suitable for our purpose.

Theorem 2.2. Let $Y$ be a d-dimensional Hilbert space with $2 \leq d<\infty$ and $\left\{\left|y_{s}\right\rangle: 0 \leq s \leq\right.$ $d-1\}$ an orthonormal basis for $Y$. Let $Z$ be the subspace $\left\{\sum_{s=0}^{d-1} \alpha_{s}\left|y_{s}\right\rangle: \sum_{s=0}^{d-1} \alpha_{s}=0\right\}$.

(i) If $d=2$ then $Z=\mathbb{C}\left(\left|y_{0}\right\rangle-\left|y_{1}\right\rangle\right)$.

(ii) Let $d \geq 3$. Then there exists an orthonormal basis $\left\{\left|z_{s}\right\rangle: 0 \leq s \leq d-2\right\}$ for $Z$ such that $\left|y_{0}\right\rangle$ occurs as a summand in $\left|z_{0}\right\rangle$ and $\left|z_{1}\right\rangle$; further, for $d>3,\left|y_{0}\right\rangle$ does not occur as a summand in $\left|z_{s}\right\rangle$ for $2 \leq s \leq d-2$.

(iii) Let $d \geq 3$. Let $1 \leq r \leq d-2$. Let

$$
Z_{r}^{1}=\left\{\sum_{s=0}^{r} \alpha_{s}\left|y_{s}\right\rangle: \sum_{s=0}^{r} \alpha_{s}=0\right\}=Z \cap\left\langle\left\{\left|y_{s}\right\rangle: 0 \leq s \leq r\right\}\right\rangle,
$$

and

$$
Z_{r}^{2}=\left\{\sum_{s=r+1}^{d-1} \alpha_{s}\left|y_{s}\right\rangle: \sum_{s=r+1}^{d-1} \alpha_{s}=0\right\} .
$$

Let $\mathcal{C}_{r}^{1}=\left\{\left|z_{s}\right\rangle: 0 \leq s \leq r-1\right\}$ be an orthonormal basis for $Z_{r}^{1}$ such that $\left|y_{0}\right\rangle$ occurs as a summand in $\left|z_{0}\right\rangle$ and in no other $\left|z_{s}\right\rangle$ for $s \leq r-1$. Then there exists an orthonormal 
basis $\left\{\left|z_{s}\right\rangle: 0 \leq s \leq d-2\right\}$ for $Z$ such that $\left|y_{0}\right\rangle$ occurs as a summand in $\left|z_{0}\right\rangle$ and $\left|z_{r}\right\rangle$ and in no other $\left|z_{s}\right\rangle$ for $0 \leq s \leq d-2$.

Proof. (i) is immediate.

(ii) Let $\left|z_{0}\right\rangle=\frac{1}{\sqrt{2}}\left(\left|y_{0}\right\rangle-\left|y_{1}\right\rangle\right),|\eta\rangle=\left(\left|y_{0}\right\rangle+\left|y_{1}\right\rangle\right)$ and $|v\rangle=\sum_{s=2}^{d-1}\left|y_{s}\right\rangle$. Set

$$
\left|z_{1}\right\rangle=\frac{(d-2)|\eta\rangle-2|v\rangle}{\sqrt{2 d(d-2)}}
$$

Then $\left|y_{0}\right\rangle$ occurs as a summand in $\left|z_{0}\right\rangle$ and $\left|z_{1}\right\rangle$.

We now consider the case $d>3$ and follow the notation in (iii). We choose any orthonormal basis for $Z_{1}^{2}$. For instance, we may choose the Fourier basis

$$
\left|z_{p}\right\rangle=\frac{1}{\sqrt{d-2}} \sum_{s=2}^{d-1} \exp \left[\frac{2 \pi \imath(s-2)(p-1)}{d-2}\right]\left|y_{s}\right\rangle, \quad 2 \leq p \leq d-2 .
$$

(iii) Let $|\eta\rangle=\sum_{s=0}^{r}\left|y_{s}\right\rangle,|v\rangle=\sum_{s=r+1}^{d-1}\left|y_{s}\right\rangle$. Consider any $|\xi\rangle=\sum_{s=0}^{d-1} \alpha_{s}\left|y_{s}\right\rangle$. For $0 \leq s^{\prime} \neq$ $s^{\prime \prime} \leq r,\left|y_{s^{\prime}}\right\rangle-\left|y_{s^{\prime \prime}}\right\rangle \in Z_{r}^{1}$. So $|\xi\rangle \perp Z_{r}^{1}$ only if $\alpha_{s^{\prime}}=\alpha_{s^{\prime \prime}}$ for $s^{\prime} \neq s^{\prime \prime}$ with $0 \leq s^{\prime} \neq s^{\prime \prime} \leq r$. Thus any such vector has the form

$$
|\xi\rangle=\alpha|\eta\rangle+\sum_{s=r+1}^{d-1} \alpha_{s}\left|y_{s}\right\rangle \quad \text { with } \quad(r+1) \alpha+\sum_{s=r+1}^{d-1} \alpha_{s}=0 .
$$

Also any $|\xi\rangle$ of the form as in $(9)$ is orthogonal to $Z_{r}^{1}$. Set

$$
\left|z_{r}\right\rangle=\frac{(d-1-r)|\eta\rangle-(r+1)|\nu\rangle}{\sqrt{d(r+1)(d-r-1)}} .
$$

Then $\left|y_{0}\right\rangle$ occurs as a summand in $\left|z_{r}\right\rangle$.

We now consider the case $r \leq d-3$, which forces $d \geq 4$ for sure. Now $|\xi\rangle$ as in (9), satisfies $\left\langle\xi \mid z_{r}\right\rangle=0$ if and only if $\alpha=0$ if and only if $\sum_{s=r+1}^{d-1} \alpha_{s}=0$ if and only if $|\xi\rangle$ has the form

$$
|\xi\rangle=\sum_{s=r+1}^{d-1} \alpha_{s}\left|y_{s}\right\rangle, \quad \sum_{s=r+1}^{d-1} \alpha_{s}=0 \quad \text { if and only if } \quad|\xi\rangle \in Z_{r}^{2} .
$$

As in the proof of (ii), we choose any orthonormal basis for $Z_{r}^{2}$. for instance, we may choose the Fourier basis,

$$
\left|z_{p}\right\rangle=\frac{1}{\sqrt{d-1-r}} \sum_{s=r+1}^{d-1} \exp \left[\frac{2 \pi(s-r-1)(p-r)}{d-1-r}\right]\left|y_{s}\right\rangle, \quad r+1 \leq p \leq d-2 .
$$

Then $\left|y_{0}\right\rangle$ does not occur as a summand in $\left|z_{p}\right\rangle, r+1 \leq p \leq d-2$.

2.10. Orthonormal basis for $\mathcal{S}$ (general case). We shall now construct a suitable orthonormal basis for $\mathcal{S}$ in our multipartite system $\mathcal{H}=\bigotimes_{j=1}^{k} \mathcal{H}_{j}$. Let $1 \leq j \neq j^{\prime} \leq k$. Set $\nu=\min \left\{d_{j}, d_{j^{\prime}}\right\}$ and $\nu^{\prime}=\max \left\{d_{j}, d_{j^{\prime}}\right\}$. We concentrate on the case $(k-2)+\left(\nu^{\prime}-\nu\right)>0$, as the remaining case $k=2, \nu=\nu^{\prime}$ comes under $\$ 2.7$ above. It is enough to construct suitable orthonormal basis for $\mathcal{S}^{(n)}$ for $1 \leq n \leq N-1$, because we can just put them together to get an orthonormal basis for $\mathcal{S}$. Let $1 \leq n \leq N-1$. We take $X=\mathcal{H}^{(n)}, Z=\mathcal{S}^{(n)}$ in the above 
theorem. We note that $\mathcal{H}^{(n)}$ has dimension $d=\left|\mathcal{I}_{n}\right|$. For $0 \leq x, x^{\prime} \leq \nu-1$, we take $\mathbf{i}^{\left(x, x^{\prime}\right)} \in \mathcal{I}$ given by

$$
i_{t}^{\left(x, x^{\prime}\right)}= \begin{cases}0 & t \neq j \text { or } j^{\prime} \\ x & t=j \\ x^{\prime} & t=j^{\prime} .\end{cases}
$$

At times we shall replace $\mathbf{i}^{\left(x, x^{\prime}\right)}$ by $\widetilde{\left(x, x^{\prime}\right)}$. For $|\xi\rangle \in \mathbb{C}^{\nu} \otimes \mathbb{C}^{\nu}$, we take $|\tilde{\xi}\rangle$ to be the vector in $\mathcal{H}$ which is obtained by considering $|\xi\rangle$ as a member of $\mathcal{H}_{j} \otimes \mathcal{H}_{j^{\prime}}$ and then filling in the remaining places by $|0\rangle$ (if any). Then $\tilde{\mathcal{B}}_{n}=\left\{|\tilde{\xi}\rangle:|\xi\rangle \in \mathcal{B}_{n}\right\}$ may be thought of as an orthonormal basis for its linear span which is a part of $\mathcal{S}^{(n)}$.

Let

$$
\begin{aligned}
& \mathcal{I}_{n}^{1}= \begin{cases}\left\{\mathbf{i} \in \mathcal{I}_{n}, 0 \leq i_{j}, i_{j^{\prime}} \leq \nu-1, \text { and } i_{t}=0 \text { for } t \neq j, j^{\prime}\right\}, & 1 \leq n \leq 2 \nu-3 \\
\emptyset & \text { otherwise }\end{cases} \\
& \mathcal{I}_{n}^{2}=\mathcal{I}_{n} \backslash \mathcal{I}_{n}^{1} .
\end{aligned}
$$

We note that $\left|\mathcal{I}_{n}^{1}\right|$ is either 0 or $\geq 2$. For $n=2 g$ with $1 \leq g \leq \nu-1$, we take $\mathbf{i}^{0}=\widetilde{(g, g)}$. For $n=2 g-1,1 \leq g \leq \nu-1$ we take $\mathbf{i}^{0}=(g-1, g)$. Next, for $1 \leq n \leq 2 \nu-3$, we arrange members of $\mathcal{I}_{n}^{1} \backslash\left\{\mathbf{i}^{0}\right\}$ in any sequence, say $\mathbf{i}^{1}, \cdots, \mathbf{i}^{\left|\mathcal{I}_{n}^{1}\right|-1}$ insisting, for $n=2 g-1, \mathbf{i}^{1}=(g, g-1)$. Then, we arrange members of $\mathcal{I}_{n}^{2}$, if any, in any manner we like. This will complete the enumeration of $\mathcal{I}_{n}$ as $0,1, \cdots,\left|\mathcal{I}_{n}\right|-1$. For $n=2 \nu-2$, we enumerate $\mathcal{I}_{n} \backslash\left\{\mathbf{i}^{0}\right\}$ as $\mathbf{i}^{1}, \cdots, \mathbf{i}^{\left|\mathcal{I}_{n}\right|-1}$. For $2 \nu-1 \leq n \leq N-1$, we enumerate $\mathcal{I}_{n}$ in any manner we like as $\mathbf{i}^{0}, \mathbf{i}^{1}, \cdots, \mathbf{i}^{\mid} \mathcal{I}_{n} \mid-1$. Finally, we set $\left|y_{s}\right\rangle=\left|\mathbf{i}^{s}\right\rangle, 0 \leq s \leq d-1=\left|\mathcal{I}_{n}\right|-1$ and, in case $1 \leq n \leq 2 \nu-3, r=\left|\mathcal{I}_{n}^{1}\right|-1$.

To distinguish constructions for different $n$ 's, we may use extra fixture $n$; for instance ${ }^{n} \mathbf{i}^{0},{ }^{n} \mathbf{i}^{1}, \cdots,\left|\eta_{n}\right\rangle,\left|v_{n}\right\rangle$ etc. in place of $\mathbf{i}^{0}, \mathbf{i}^{1}, \cdots,|\eta\rangle,|v\rangle$.

This discussion combined with Theorem 2.2 above immediately gives us the following theorem.

Theorem 2.3. Let $\mathcal{H}=\bigotimes_{t=1}^{k} \mathcal{H}_{t}$. Let $1 \leq j \neq j^{\prime} \leq k, \nu=\min \left\{d_{j}, d_{j^{\prime}}\right\} \leq \nu^{\prime}=\max \left\{d_{j}, d_{j^{\prime}}\right\}$ and $(k-2)+\left(\nu^{\prime}-\nu\right)>0$. There exists an orthonormal basis $\mathcal{C}$ for $\mathcal{S}$ such that

(i) $|0\rangle \otimes|0\rangle$ does not occur as a summand in any vector in $\mathcal{C}$.

(ii) For $1 \leq g \leq \nu-2, \mid \widetilde{|g\rangle \otimes \mid}$ g $\rangle$ occurs as a summand in two members of $\mathcal{C}$.

(iii) $(|\nu-1\rangle \otimes|\nu-1\rangle)$ occurs as a summand in two members of $\mathcal{C}$ except for the bipartite case with $2=\nu<\nu^{\prime}$ or $\nu^{\prime}=\nu+1$, when it occurs only once.

(iv) For $2 \leq g \leq \nu-1,(|g-\widehat{1\rangle \otimes}| g\rangle)$ and $(|g\rangle \widehat{\otimes|g-1\rangle})$ occur as a summand in (the same) two members of $\mathcal{C}$.

(v) In particular, $(|\widehat{|0\rangle \otimes \mid}\rangle),(|\widehat{|1\rangle \otimes \mid} 0\rangle)$ and $(\mid \widehat{|1\rangle \otimes|1\rangle})$, occur as summands as follows.

(a) Vectors $|0\rangle \otimes|1\rangle$ and $\mid \widehat{|1\rangle \otimes|0\rangle}$ occur as a summand in $\widetilde{\left|a_{0,1}\right\rangle}=\frac{1}{\sqrt{2}}(|\widehat{0\rangle \otimes|1\rangle}-| \widehat{1\rangle \otimes|0\rangle})$, and in case $k \geq 3$, also in $\left|c_{0}^{1}\right\rangle=\frac{1}{\sqrt{2\left|\mathcal{I}_{1}\right|\left(\left|\mathcal{I}_{1}\right|-2\right)}}\left(\left(\left|\mathcal{I}_{1}\right|-2\right)(|\widehat{0\rangle \otimes|1\rangle}+| \widehat{1\rangle \otimes|0\rangle})-2\left|v_{1}\right\rangle\right)=$ $\frac{1}{\sqrt{2 k(k-2)}}\left((k-2)(|0\rangle \otimes|1\rangle+\mid \widehat{1\rangle \otimes|0\rangle})-2\left|v_{1}\right\rangle\right)$.

(b) For $\nu=2,(\mid \widehat{1\rangle \otimes|1\rangle})$ occurs as a summand as follows. 
- For $k=2, \nu^{\prime} \geq 3$, in $\frac{1}{\sqrt{2}}(|\widehat{1\rangle \otimes|1\rangle}-| \widehat{0\rangle \otimes|2\rangle})$ or in $\frac{1}{\sqrt{2}}(|\widehat{1\rangle \otimes|1\rangle}-| \widehat{2\rangle \otimes|0\rangle})$ according as $d_{2}=\nu^{\prime}$ or $d_{1}=\nu^{\prime}$. In fact, it is the same as $\left|a_{2} \mathbf{i}^{0}, \mathbf{i}^{1}\right\rangle=\frac{1}{\sqrt{2}}\left(\left.\right|^{2} \mathbf{i}^{0}\right\rangle-$ $\left.\left|{ }^{2} \mathbf{i}^{1}\right\rangle\right)$.

- For $k \geq 3$, in $\left|a_{2_{\mathbf{i}^{0}},{ }_{2} \mathbf{i}^{1}}\right\rangle=\frac{1}{\sqrt{2}}\left(\left|{ }^{2} \mathbf{i}^{0}\right\rangle-\left|{ }^{2} \mathbf{i}^{1}\right\rangle\right)$ and in $\left|c_{0}^{2}\right\rangle=\frac{\left(\left|\mathcal{I}_{2}\right|-2\right)\left(\left|{ }^{2} \mathbf{i}^{0}\right\rangle+\left|{ }^{2} \mathbf{i}^{1}\right\rangle\right)-2\left|v_{2}\right\rangle}{\sqrt{2\left(\left|\mathcal{I}_{2}\right|-2\right)\left|\mathcal{I}_{2}\right|}}$

(c) For $\nu \geq 3, \mid \widetilde{1\rangle \otimes|1\rangle}$ occurs as a summand in $\left|\tilde{b_{0}^{2}}\right\rangle$, and if, in addition, $k \geq 3$, also in $\left|c_{0}^{2}\right\rangle=\frac{\left.\mathcal{I}_{2}^{2}|| \eta_{2}\right\rangle-\left|\mathcal{I}_{2}^{1}\right|\left|v_{2}\right\rangle}{\sqrt{\left|\mathcal{I}_{2}^{1}\right|\left|\mathcal{I}_{2}^{2}\right|\left|\mathcal{I}_{2}\right|}}$

\section{Entanglement PRoperties of the PRojeCtion operators}

We begin this section with some preparatory remarks, which will be used to arrive at our main results.

3.1. A useful involution on $\mathcal{I} \times \mathcal{I}$. Let $\mathcal{H}=\bigotimes_{t=1}^{k} \mathcal{H}_{t}$. Fix $j$, with $1 \leq j \leq k$.

For $(\mathbf{p}, \mathbf{q}) \in \mathcal{I} \times \mathcal{I}$, let $\sigma_{j}(\mathbf{p}, \mathbf{q})=\left(\mathbf{p}^{\prime}, \mathbf{q}^{\prime}\right)$, where

$$
p_{t}^{\prime}=\left\{\begin{array}{ll}
p_{t} & \text { for } t \neq j \\
q_{j} & \text { for } t=j
\end{array} \quad \text { and } \quad q_{t}^{\prime}= \begin{cases}q_{t} & \text { for } t \neq j \\
p_{j} & \text { for } t=j\end{cases}\right.
$$

Then

$$
|\mathbf{p}\rangle\left\langle\left.\mathbf{q}\right|^{P T_{j}}=\mid \mathbf{p}^{\prime}\right\rangle\left\langle\mathbf{q}^{\prime}\right|
$$

We note that $\sigma_{j}(\mathbf{q}, \mathbf{p})=\left(\mathbf{q}^{\prime}, \mathbf{p}^{\prime}\right)$. Further, the map $\sigma_{j} \circ \sigma_{j}$ is the identity map on $\mathcal{I} \times \mathcal{I}$, i.e., the map $\sigma_{j}$ is an involution on $\mathcal{I} \times \mathcal{I}$.

3.2. Action of $P T_{j}$. Any operator $\rho \in \mathcal{L}\left(\mathcal{H}_{1} \otimes \cdots \otimes \mathcal{H}_{k}\right)$ given as in $(2)$ can be written in the compact form as,

$$
\rho=\sum_{\mathbf{p}, \mathbf{q} \in \mathcal{I}} \rho_{(\mathbf{p}, \mathbf{q})}|\mathbf{p}\rangle\langle\mathbf{q}|
$$

then

$$
\begin{aligned}
\rho^{P T_{j}} & =\sum_{(\mathbf{p}, \mathbf{q}) \in \mathcal{I} \times \mathcal{I}} \rho_{(\mathbf{p}, \mathbf{q})}\left|\mathbf{p}^{\prime}\right\rangle\left\langle\mathbf{q}^{\prime}\right| \\
& =\sum_{(\mathbf{p}, \mathbf{q}) \in \mathcal{I} \times \mathcal{I}} \rho_{\sigma_{j}(\mathbf{p}, \mathbf{q})}|\mathbf{p}\rangle\langle\mathbf{q}| .
\end{aligned}
$$

Fix $j^{\prime} \neq j$ with $1 \leq j^{\prime} \leq k$. Let $\mathbf{p}^{0} \in \mathcal{I}_{0}, \mathbf{q}^{0} \in \mathcal{I}_{2} ; \mathbf{p}^{1}$ and $\mathbf{q}^{1} \in \mathcal{I}_{1}$, be defined as

$$
\begin{array}{cc}
p_{t}^{0}=0 \quad \text { for all } t & q_{t}^{0}= \begin{cases}1 & \text { for } t=j, j^{\prime} \\
0 & \text { otherwise }\end{cases} \\
p_{t}^{1}=\left\{\begin{array}{ll}
1 & \text { for } t=j \\
0 & \text { otherwise }
\end{array} \quad, \quad q_{t}^{1}= \begin{cases}1 & \text { for } t=j^{\prime} \\
0 & \text { otherwise }\end{cases} \right.
\end{array}
$$

Then $\sigma_{j}\left(\mathbf{p}^{0}, \mathbf{q}^{0}\right)=\left(\mathbf{p}^{1}, \mathbf{q}^{1}\right)$. 
ENTANGLEMENT PROPERTIES OF POSITIVE OPERATORS WITH RANGES IN COMPLETELY ENTANGLED SUBSPAC

Let $\lambda \neq 0$ be a real number. Set $|\xi\rangle=\lambda\left|\mathbf{p}^{0}\right\rangle+\left|\mathbf{q}^{0}\right\rangle$. Then for any $\mathbf{p}, \mathbf{q} \in \mathcal{I}$,

$$
\begin{aligned}
\langle\xi \mid \mathbf{p}\rangle\langle\mathbf{q} \mid \xi\rangle= & \left(\lambda \delta_{\mathbf{p}^{0} \mathbf{p}}+\delta_{\mathbf{q}^{0} \mathbf{p}}\right)\left(\lambda \delta_{\mathbf{p}^{0} \mathbf{q}}+\delta_{\mathbf{q}^{0} \mathbf{q}}\right) \\
= & \left\{\begin{array}{lll}
\lambda^{2} & \text { for } & (\mathbf{p}, \mathbf{q})=\left(\mathbf{p}^{\mathbf{0}}, \mathbf{p}^{\mathbf{0}}\right), \\
\lambda & \text { for } & (\mathbf{p}, \mathbf{q}) \in\left\{\left(\mathbf{p}^{\mathbf{0}}, \mathbf{q}^{\mathbf{0}}\right),\left(\mathbf{q}^{\mathbf{0}}, \mathbf{p}^{\mathbf{0}}\right)\right\} \\
1 & \text { for } & (\mathbf{p}, \mathbf{q})=\left(\mathbf{q}^{\mathbf{0}}, \mathbf{q}^{\mathbf{0}}\right) \\
0 & & \text { otherwise }
\end{array}\right.
\end{aligned}
$$

With a state $\rho$ as in 11,

$$
\begin{aligned}
\left\langle\xi\left|\rho^{P T_{j}}\right| \xi\right\rangle & =\sum_{(\mathbf{p}, \mathbf{q}) \in \mathcal{I} \times \mathcal{I}} \rho_{\sigma_{j}(\mathbf{p}, \mathbf{q})}\langle\xi \mid \mathbf{p}\rangle\langle\mathbf{q} \mid \xi\rangle \\
& =\lambda^{2} \rho_{\sigma_{j}\left(\mathbf{p}^{\mathbf{0}}, \mathbf{p}^{\mathbf{0}}\right)}+\lambda \rho_{\sigma_{j}\left(\mathbf{p}^{\mathbf{0}}, \mathbf{q}^{\mathbf{0}}\right)}+\lambda \rho_{\sigma_{j}\left(\mathbf{q}^{\mathbf{0}}, \mathbf{p}^{\mathbf{0}}\right)}+\rho_{\sigma_{j}\left(\mathbf{q}^{0}, \mathbf{q}^{0}\right)} \\
& =\lambda^{2} \rho_{\left(\mathbf{p}^{0}, \mathbf{p}^{0}\right)}+\lambda\left(\rho_{\left(\mathbf{p}^{1}, \mathbf{q}^{1}\right)}+\rho_{\left(\mathbf{q}^{1}, \mathbf{p}^{1}\right)}\right)+\rho_{\left(\mathbf{q}^{0}, \mathbf{q}^{\mathbf{0}}\right)} .
\end{aligned}
$$

Theorem 3.1. Let $\mathcal{H}=\bigotimes_{r=1}^{k} \mathcal{H}_{r}$. Let $P_{\mathcal{S}}$ be the projection on the completely entangled subspace $\mathcal{S}$. For each $j, P_{\mathcal{S}}$ is not positive under partial transpose at level $j$.

In particular, $P_{\mathcal{S}}$ is $N P T$.

Proof. For a unit vector $|\zeta\rangle \in \mathcal{H}$, let $P_{\zeta}$ be the projection on $|\zeta\rangle$, i.e. $P_{\zeta}=|\zeta\rangle\langle\zeta|$. Let $1 \leq j \leq k$. Take any $j^{\prime} \neq j$ with $1 \leq j^{\prime} \leq k$. Let $\mathcal{C}$ be an orthonormal basis for $\mathcal{S}$ in two separate cases as follows.

(a) For $k=2, d_{1}=d_{2}=\nu$, take $\mathcal{C}=\mathcal{B}$ as in $\$ 2.7$.

(b) For $k=2$ but $d_{1} \neq d_{2}$, or $k \geq 3$ we follow the procedure set up in $\$ 2.10$ for Theorem 2.3 . Then

$$
P_{\mathcal{S}}=\sum_{|\zeta\rangle \in \mathcal{C}} P_{\zeta}=\sum_{\mathbf{p}, \mathbf{q} \in \mathcal{I}} \rho_{(\mathbf{p}, \mathbf{q})}|\mathbf{p}\rangle\langle\mathbf{q}|
$$

for some suitable $\rho_{(\mathbf{p}, \mathbf{q})}$ 's. In the notation $\$ 3.2$.

$$
\left\langle\xi\left|\rho^{P T_{j}}\right| \xi\right\rangle=\lambda^{2} \rho_{\left(\mathbf{p}^{\mathbf{0}}, \mathbf{p}^{\mathbf{0}}\right)}+\lambda\left(\rho_{\left(\mathbf{p}^{\mathbf{1}}, \mathbf{q}^{\mathbf{1}}\right)}+\rho_{\left(\mathbf{q}^{1}, \mathbf{p}^{\mathbf{1}}\right)}\right)+\rho_{\left(\mathbf{q}^{\mathbf{0}}, \mathbf{q}^{\mathbf{0}}\right)} .
$$

To complete the proof it is enough to show that $\left\langle\xi\left|\rho^{P T_{j}}\right| \xi\right\rangle<0$.

We arrange the elements of $\mathcal{C}$ in any manner $\left\{\left|\zeta_{s}\right\rangle: 0 \leq s \leq M-1\right\}$, but insisting on the following points.

(c)

(d)

$$
\left|\zeta_{0}\right\rangle= \begin{cases}\left|a_{0,1}\right\rangle & \text { in case (a) } \\ \widetilde{\left|a_{0,1}\right\rangle} & \text { in case (b). }\end{cases}
$$

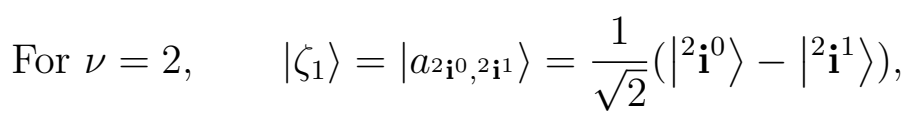

$$
\begin{aligned}
& \text { whereas for } \nu \geq 3, \quad\left|\zeta_{1}\right\rangle=\widetilde{\left|b_{0}^{2}\right\rangle} \text {. }
\end{aligned}
$$

$$
\text { For } k \geq 3, \quad\left|\zeta_{2}\right\rangle=\left|c_{0}^{1}\right\rangle \text {. }
$$

For $k \geq 3, \quad\left|\zeta_{3}\right\rangle=\left|c_{0}^{2}\right\rangle$. 
We write $P_{s}=P_{\left|\zeta_{s}\right\rangle}, 0 \leq s \leq M-1$. Then $P_{\mathcal{S}}=\sum_{s=0}^{M-1} P_{s}$. So $\rho_{\mathbf{p}, \mathbf{q}} \neq 0$ only if $|\mathbf{p}\rangle$ and $|\mathbf{q}\rangle$ occur as a summand in some $\left|\zeta_{s}\right\rangle$.

In view of Theorem 2.3(iv) and (12) above we can just confine our attention to the vectors listed under (c), (d), (e) and (f) above.

We first note that none of them contributes towards $\rho_{\left(\mathbf{p}^{\mathbf{0}}, \mathbf{p}^{\mathbf{0}}\right)}$. Also $\rho_{\left(\mathbf{q}^{\mathbf{0}}, \mathbf{q}^{\mathbf{0}}\right)} \geq 0$. Next, we find that contribution to $\rho_{\left(\mathbf{p}^{1}, \mathbf{q}^{1}\right)}$ is the same as that to $\rho_{\left(\mathbf{q}^{1}, \mathbf{p}^{1}\right)}$. Thus, if the final contribution to $\rho_{\left(\mathbf{p}^{\mathbf{1}}, \mathbf{q}^{\mathbf{1}}\right)}$ is $<0$, then for a suitable $\lambda>0,\left\langle\xi\left|\rho^{P T_{j}}\right| \xi\right\rangle<0$. We now proceed to show that it is so.

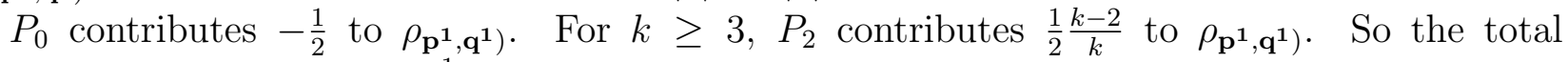
contribution to $\rho_{\left(\mathbf{p}^{1}, \mathbf{q}^{\mathbf{1}}\right)}$ is $-\frac{1}{k}$. Hence the proof.

Corollary 3.1. $\mathcal{F}$ does not contain any unextendable orthonormal product basis.

Proof. If $\mathcal{F}$ contains any unextandable product basis then by Theorem (A) $P_{\mathcal{S}}$ will be PPT which is not true by Theorem 3.1. Hence the result follows.

We now show that large classes of states with range in the completely entangled subspace $\mathcal{S}$ are NPT.

Theorem 3.2. Let $1 \leq j \leq k$. Take any $j^{\prime} \neq j$ with $1 \leq j^{\prime} \leq k$. Any positive operator $\sum_{s=0}^{M-1} p_{s} P_{s}$, where $p_{s} \geq 0$ for all $s, p_{0}+(k-2) p_{2}>0$ and $P_{s}$ 's are as in the proof of Theorem 3.1 above, is not positive under partial transpose at level $j$.

Proof. (i) All cases except possibly the case when $k \geq 3$ and $(k-2) p_{2}=k p_{0}$.

We refer to the proof of Theorem 3.1 above. The only change needed is that the term, say $w$ with $\lambda$ is now given as follows.

(a) For $k=2, w=-p_{0}$ (in place of -1 ),

(b) In case $k \geq 3, w=-p_{0}+p_{2} \frac{k-2}{k} \neq 0$.

So the final number in the right hand side of $(12)$ can be made negative by suitable choice of $\lambda$ which has to be suitably big and $>0$ if $w<0$, and has to be $<0$ and suitably big in absolute value if $w>0$.

(ii) Case $k \geq 3$ but $(k-2) p_{2}=k p_{0}$. Since $p_{0}+(k-2) p_{2}>0$, we have $p_{2}>0$. Because $k \geq 3$, there is $j^{\prime \prime}$ with $j \neq j^{\prime \prime} \neq j^{\prime}$ and $1 \leq j^{\prime \prime} \leq k$. Let $\mathbf{r}^{0} \in \mathcal{I}_{2}$ and $\mathbf{r}^{1} \in \mathcal{I}_{1}$ be given by

$$
\begin{gathered}
r_{t}^{0}=\left\{\begin{array}{lll}
1 & \text { if } & t=j, j^{\prime \prime} \\
0 & & \text { otherwise }
\end{array}\right. \\
r_{t}^{1}=\left\{\begin{array}{lll}
1 & \text { for } & t=j^{\prime \prime} \\
0 & & \text { otherwise. }
\end{array}\right.
\end{gathered}
$$

We replace $\xi$ by $\xi^{\prime}$ given by $\lambda\left|\mathbf{p}^{0}\right\rangle+\left|\mathbf{r}^{0}\right\rangle$ with $\lambda$ real and make computations similar to those in item 3.2 and proof of part (i) above. We note that $\mathbf{q}^{1}$ has to be replaced by $\mathbf{r}^{1}$, and then $w$ by $w^{\prime}=-\frac{2}{k} p_{2}$. And, therefore, for $\lambda$ suitably bigger than $0,\left\langle\xi^{\prime}\left|\rho^{P T_{j}}\right| \xi^{\prime}\right\rangle<0$. This completes the proof.

\section{Remark 3.1.}

(i) Because of the freedom of orthonormal bases at various stages of the construction of $\mathcal{C}$ the import of Theorem 3.2 is much more. In fact, we may apply Theorem 2.2 to construct a basis $\mathcal{D}$ for $\mathcal{S}$ with more such freedom by clubbing in $\mathcal{S}^{(n)}$ 's, $3 \leq n \leq N-1$ and insisting on including $\left|\zeta_{0}\right\rangle,\left|\zeta_{1}\right\rangle$, and in case $k \geq 3,\left|\zeta_{2}\right\rangle$ and $\left|\zeta_{3}\right\rangle$ as well. 
(ii) Let $1 \leq r \leq k$. Let $\gamma_{r}$ be the involution on the set $\mathcal{D}_{r}=\left\{p: 0 \leq p \leq d_{r}-1\right\}$ to itself that takes $p \mapsto d_{r}-1-p$ for $0 \leq p \leq d_{r}-1$. This induces a unitary linear operator $R_{r}$ on $\mathcal{H}_{r}$ to itself which takes $e_{p}$ to $e_{\gamma_{r}(p)}$ for $p \in \mathcal{D}_{r}$. We note that $R_{r}^{2}=I_{\mathcal{H}_{r}}$ and therefore, $R_{r}$ is self-adjoint. Next, let $\gamma=\prod_{r=1}^{k} \gamma_{r}$ on $\mathcal{I}=\prod_{r=1}^{k} \mathcal{D}_{r}$ to itself. Then $\gamma$ is an involution on $\mathcal{I}$ to itself. Further, for $0 \leq n \leq N, \gamma$ takes $\mathcal{I}_{n}$ to $\mathcal{I}_{N-n}$. Let $R$ be the operator $\bigotimes_{r=1}^{k} R_{r}$ on $\mathcal{H}$ to itself. Then, for $0 \leq n \leq N, R$ takes $\mathcal{H}^{(n)}$ onto $\mathcal{H}^{(N-n)}, u_{n}$ to $u_{N-n}$, $\mathcal{T}^{(n)}$ onto $\mathcal{T}^{(N-n)}, \mathcal{S}^{(n)}$ onto $\mathcal{S}^{(N-n)}$. Therefore, $R$ takes $\mathcal{S}$ onto itself. Further, $R$ is unitary and self-adjoint. For $\mathbf{p}, \mathbf{q} \in \mathcal{I}, R(|\mathbf{p}\rangle\langle\mathbf{q}|) R=|\gamma(\mathbf{p})\rangle\langle\gamma(\mathbf{q})|$. Also, for $1 \leq j \neq j^{\prime} \leq k$, we may now consider $R^{\dagger} \rho R=R \rho R$ with $\rho$ 's as indicated in Theorem 3.2 and part (i) above to add to the class of positive operators with range in $\mathcal{S}$ whose partial transpose at level $j$ is not positive.

(iii) For $1 \leq j \leq k$ and $1 \leq j^{\prime} \leq k$ with $j \neq j^{\prime}$ let $\mathcal{N}_{j, j^{\prime}}$ be the set of $N P T_{j}$ states obtained in Theorem 3.2 together with those by methods indicated in (i) and (ii) above. Put

$$
\mathcal{N}=\bigcup_{\substack{1 \leq j \leq k \\ 1 \leq j^{\prime} \leq k \\ j \neq j^{\prime}}} \mathcal{N}_{j, j^{\prime}} .
$$

Then each $\rho$ in $\mathcal{N}$ has range in the subspace $\mathcal{S}$ and has a non-positive partial transpose at some level.

(iv) Johnston [Joh13] asked the following question.

What is the maximum dimension $\mu$ of a subspace with the property that any state with range in the subspace has at least one partial transpose which is non-positive.

Let us call a subspace $\mathcal{E}$ of $\mathcal{H}$ satisfying this criteria an NPT space.

(v) Let $\mathcal{E}$ be a subspace of $\mathcal{H}$. If $\{\rho: \rho$ is a state with range in $\mathcal{E}\}$ is contained in $\mathcal{N}$, then $\mathcal{E} \subset \mathcal{S}$ and $\mathcal{E}$ is $N P T$. In particular, If $\mathcal{N}=\{\rho: \rho$ is a state with range in $\mathcal{S}\}$, then $\mathcal{S}$ is NPT. If that be so, then the answer to Johnston's question is

$$
\mu=M=d_{1} d_{2} \cdots d_{k}-\left(d_{1}+d_{2}+\cdots+d_{k}\right)+k-1 .
$$

This question still remains open, but the progress made in this paper above does show that $\mathcal{N}$ is substancially large.

\section{COnClusion}

Let $\mathcal{S}$ be a concrete completely entangled subspace of maximal dimension, in $\mathcal{H}=\bigotimes_{j=1}^{k} \mathcal{H}_{j}$ with $2 \leq d_{j}=\operatorname{dim} \mathcal{H}_{j}<\infty$ for $1 \leq j \leq k$, constructed by Parthasarathy [Par04]. Let $P_{\mathcal{S}}$ be the projection on this space. We realized that the particular orthonormal basis $\mathcal{B}$ for $\mathcal{S}$ for the bipartite case of equal dimensions obtained by Parthasarathy [Par04] helps us to prove that $P_{\mathcal{S}}$ is not positive under partial transpose. For any fixed $j$ and $j^{\prime}$ with $1 \leq j \neq j^{\prime} \leq k$, we developed techniques to construct a suitable orthonormal basis $\mathcal{C}$ for $\mathcal{S}$ for the multipartite case utilizing $\mathcal{B}$ in the process. This enabled us to prove that $P_{\mathcal{S}}$ is not positive under partial transpose at level $j$. We next extended this to certain positive operators $\rho$ 's with range contained in $\mathcal{S}$. This generalizes a substantial part of the corresponding result of Johnston [Joh13] for the bipartite case. Even after varying $j$ and $j^{\prime}$ and clubbing all $\rho$ 's, the question whether there are any states with support in $\mathcal{S}$ that are $\mathrm{PPT}_{j}$ for each $j, 1 \leq j \leq k$, remains open. However, in this paper we have made substantial progress in the direction of obtaining an answer. Further results on this issue will be presented elsewhere. 


\section{REFERENCES}

[BDM $\left.{ }^{+} 99\right]$ Charles H. Bennett, David P. DiVincenzo, Tal Mor, Peter W. Shor, John A. Smolin, and Barbara M. Terhal. Unextendible product bases and bound entanglement. Phys. Rev. Lett., 82(26):5385-5388, Jun 1999.

[Bha06] B. V. Rajarama Bhat. a completely entangled subspace of maximal dimension. Int. J. Quantum Inf., 4(2):325-330, 2006.

[Cho80] Man Duen Choi. Positive linear maps. In Operator Algebras and Applications (Kingston, 1980), Proceedings of the Symposium on Pure Mathematics, volume 38 (2), pages 583 - 590. American Mathematical Society, 1980.

$\left[\mathrm{DMS}^{+} 03\right]$ David P. DiVincenzo, Tal Mor, Peter W. Shor, John A. Smolin, and Barbara M. Terhal. Unextendible product bases, uncompletable product bases and bound entanglement. Comm. Math. Phys., 238(3):379-410, 2003.

[EPR35] A. Einstein, B. Podolsky, and N. Rosen. Can quantum-mechanical description of physical reality be considered complete? Phys. Rev., 47:777-780, May 1935.

[HHHH09] Ryszard Horodecki, Paweł Horodecki, Michał Horodecki, and Karol Horodecki. Quantum entanglement. Rev. Mod. Phys., 81(2):865-942, Jun 2009.

[Joh13] Nathaniel Johnston. Non-positive-partial-transpose subspaces can be as large as any entangled subspace. Phys. Rev. A, 87:064302, Jun 2013.

[Joh14] N. Johnston. The Structure of Qubit Unextendible Product Bases. ArXiv e-prints, January 2014, 1401.7920 .

[NC10] Michael A. Nielsen and Isaac L. Chuang. Quantum Computation and Quantum Information. Cambridge University Press, 10th anniversary edition, 2010. Cambridge Books Online.

[Par04] K. R. Parthasarathy. On the maximal dimension of a completely entangled subspace for finite level quantum systems. Proc. Indian Acad. Sci. Math. Sci., 114(4):365-374, 2004.

[Par06] K. R. Parthasarathy. Quantum computation, quantum error correcting codes and information theory. Published for the Tata Institute of Fundamental Research, Mumbai, 2006.

[Per96] Asher Peres. Separability criterion for density matrices. Phys. Rev. Lett., 77(8):1413-1415, Aug 1996.

[Sch35] E. Schrödinger. Discussion of probability relations between separated systems. Mathematical Proceedings of the Cambridge Philosophical Society, 31:555-563, 101935.

[Sch36] E. Schrödinger. Probability relations between separated systems. Mathematical Proceedings of the Cambridge Philosophical Society, 32:446-452, 101936.

[Stø82] Erling Størmer. Decomposable positive maps on $C^{*}$-algebras. Proc. Amer. Math. Soc., 86(3):402404, 1982.

[Wal02] Nolan R. Wallach. An unentangled Gleason's theorem. In Quantum computation and information (Washington, DC, 2000), volume 305 of Contemp. Math., pages 291-298. Amer. Math. Soc., Providence, RI, 2002.

[Wil13] Mark M. Wilde. Quantum information theory. Cambridge University Press, Cambridge, 2013.

[Wor76] S. L. Woronowicz. Positive maps of low dimensional matrix algebras. Rep. Math. Phys., 10(2):165183, 1976.

Theoretical Statistics and Mathematics Unit, Indian Statistical Institute, Delhi Centre, 7 S J S Sansanwal Marg, New Delhi 110 016, India

E-mail address, R Sengupta: rb@isid.ac.in, ritabrata.sengupta@gmail.com

Department of Physical Sciences, Indian Institute of Science Education \& Research, Mohali; Sector 81, Knowledge City, Mohali 140 306, India

E-mail address, Arvind: arvind@iisermohali.ac.in

Theoretical Statistics and Mathematics Unit, Indian Statistical Institute, Delhi Centre, 7 S J S Sansanwal Marg, New Delhi 110 016, India

E-mail address, Ajit Iqbal Singh: aisingh@isid.ac.in, ajitis@gmail.com 